\section{A General Theorem for Degree-Reduction of a Digital BR Function}

\section{P. P. VAIDYANATHAN}

\begin{abstract}
A general theorem is developed for the degree reduction of a bounded real (BR) digital filter transfer function, and is related to the extraction of a digital lossless two-pair from a BR function in such a manner that the remainder function is reduced order BR. The theorem finds applications in the synthesis of digital filter transfer functions in the form of a cascade of lossless two pairs.
\end{abstract}

A general procedure for the synthesis of a digital filter transfer function in the form of a cascade of lossless two-pairs has recently been advanced [1]. The structures that result from such a synthesis have excellent passband sensitivity, and exhibit several other desirable properties under a finite-wordlength environment. The key step in the synthesis procedures in [1] is the extraction of a lossless two-pair from a bounded real (BR) transfer function in such a manner that the remainder function is a reduced order BR function. Only first- and second-order two-pair extractions were considered in [1], and no explicit proof of the BR property of the remainder function was included, even though a proof can be found in [2].

The purpose of this correspondence is to present a general theorem, which deals with the extraction of an $n$ th-order lossless two-pair from an $m$ th-order BR function. The theorem specifies the conditions under which such an extraction leads to a reduced-order BR remainder. The proof of the theorem automatically serves as a proof for the claims made in [1] concerning BR nature of the remainder function.

We begin by reviewing a few basic concepts. A transfer function $G(z)$ is said to be BR if it is stable, real-valued for real $z$ and $\left|G\left(e^{j \omega}\right)\right| \leqslant 1$ for all $\omega$. Note that in this correspondence "stable" stands for bounded-input bounded-output stability (i.e., all poles are strictly inside the unit circle). A digital two-pair (Fig. $1)$, described either by the chain parameters $(A, B, C, D)$ :

$$
\left[\begin{array}{l}
X_{1}(z) \\
Y_{1}(z)
\end{array}\right]=\left[\begin{array}{ll}
A(z) & B(z) \\
C(z) & D(z)
\end{array}\right]\left[\begin{array}{l}
Y_{2}(z) \\
X_{2}(z)
\end{array}\right]
$$

or by the transfer parameter matrix $\tau(z)=\left[T_{i j}(z)\right]$ :

$$
\left[\begin{array}{l}
Y_{1}(z) \\
Y_{2}(z)
\end{array}\right]=\left[\begin{array}{ll}
T_{11}(z) & T_{12}(z) \\
T_{21}(z) & T_{22}(z)
\end{array}\right]\left[\begin{array}{l}
X_{1}(z) \\
X_{2}(z)
\end{array}\right]
$$

is said to be Lossless Bounded Real (LBR) is each entry $T_{i j}(z)$ is $\mathrm{BR}$ and the following condition holds:

$$
\tau^{\prime}\left(z^{-1}\right) \tau(z)=I .
$$

The condition of (3), called paraunitariness, is equivalent to the following set of conditions in terms of the chain parameters:

$$
\tilde{A} A=1+\tilde{C} C, \quad \tilde{D} D=1+\tilde{B} B, \quad \tilde{C} D=\tilde{A} B .
$$

Here the "tilde" accent is defined as follows: $\tilde{A}(z)=A\left(z^{-1}\right)$, and so on. Thus on the unit circle of the $z$-plane an LBR two-pair

Manuscript received November 13,1984 . This work was supported in part by the NSF Grant ECS 84-04245.

The author is with the Department of Electrical Engineering, California Institute of Technology, Pasadena, CA 91125.

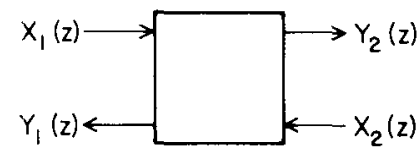

Fig. 1. The digital two-pair.

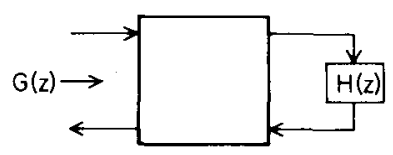

Fig. 2. Digital two-pair extraction.

satisfies

$$
|A|^{2}=1+|C|^{2}, \quad|D|^{2}=1+|B|^{2}, \quad C^{*} D=A^{*} B .
$$

The $T_{i j}$ parameters are related to the chain parameter as

$$
T_{11}=\frac{C}{A}, \quad T_{12}=\frac{A D-B C}{A}, \quad T_{21}=\frac{1}{A}, \quad T_{22}=-\frac{B}{A} .
$$

Given a BR transfer function $G(z)=P(z) / Q(z)$, let us assume that an LBR two-pair has been extracted (Fig. 2), giving rise to a remainder $H(z)$. The functions $G(z)$ and $H(z)$ are related by

$$
G(z)=\frac{P(z)}{Q(z)}=\frac{C(z)+D(z) H(z)}{A(z)+B(z) H(z)} .
$$

We first state the following result:

Lemma 1. Let $G(z)$ be BR and let the extracted two-pair LBR. Then $H(z)$ in (7) is such that $\left|H\left(e^{j \omega}\right)\right| \leqslant 1$ for all $\omega$.

Proof: From (7) we have on the unit circle,

$$
\begin{aligned}
|G|^{2}\left[|A|^{2}+|B|^{2}|H|^{2}+A B^{*} H^{*}+A^{*} B H\right] \\
=\left[|C|^{2}+|D|^{2}|H|^{2}+C D^{*} H^{*}+C^{*} D H\right] .
\end{aligned}
$$

But the LBR two-pair satisfies (5) on the unit circle. Moreover, $G(z)$ being BR, we have $|G|^{2} \leqslant 1$ on the unit circle. Accordingly, (8) implies

$$
|A|^{2}+|B|^{2}|H|^{2} \geqslant|C|^{2}+|D|^{2}|H|^{2}
$$

which reduces to

$$
|H|^{2} \leqslant 1, \quad \text { for }|z|=1
$$

with the use of (5). This proves the lemma.

Without loss of generality, we will assume the chain parameters to be polynomials in $z$. Assume that $G(z)$ is $m$ th-order BR, i.e.,

$$
G(z)=\frac{P(z)}{Q(z)}=\frac{p_{0} z^{m}+p_{1} z^{m-1}+\cdots+p_{m}}{z^{m}+q_{1} z^{m-1}+\cdots+q_{m}}
$$

with $q_{m} \neq 0$, and furthermore, $P(z)$ and $Q(z)$ are relatively prime so that there are no cancelling factors in (11). Let the polynomial $A(z)$ be the least common multiple (LCM) of all the denominators in the $T_{i j}$ parameters (see (6)), and let $n$ denote its degree. Let $H(z)$ be of the form

$$
H(z)=\frac{R(z)}{S(z)}=\frac{r_{0} z^{k}+r_{1} z^{k-1}+\cdots+r_{k}}{z^{k}+s_{1} z^{k-1}+\cdots+s_{k}}
$$


with $s_{k} \neq 0$. We now claim that if $H(z)$ is of degree $k=m-n$, then it is indeed BR. In order to prove this, it only remains to prove that $H(z)$ is stable because, by Lemma $1 ; H(z)$ already satisfies (10). Now (7) can be rewritten as

$$
G(z)=\frac{P(z)}{Q(z)}=\frac{C(z) S(z)+D(z) R(z)}{A(z) S(z)+B(z) R(z)} .
$$

Notice that the degree of $B(z)$ cannot exceed that of $A(z)$ (otherwise $T_{22}(z)$, which is BR, would not be bounded at infinity). Furthermore, the degree of $R(z)$ does not exceed that of $S(z)$. As a result, the degree of $Q(z)$ is almost equal to the sum of degrees of $A(z)$ and $S(z)$. If the degree of $H(z)$ is indeed $m-n$, then the above-montioned sum is precisely $m$ which is the degree of $Q(z)$. Therefore, there cannot be any cancellation of factors between $C(z) S(z)+D(z) R(z)$ and $A(z) S(z)+$ $B(z) R(z)$ in (13). Thus, if $H(z)$ has degree $m-n$, then $Q(z)$ is precisely given by

$$
Q(z)=A(z) S(z)+B(z) R(z) .
$$

Since $G(z)$ is BR and $\tau(z) \mathrm{LBR}, Q(z)$ and $A(z)$ have no zeros on or outside the unit circle. Next, all the functions in (14) are polynomials, and are analytic on and inside the unit circle. Moreover, the following equality holds on the unit circle:

$$
|A(z) S(z)| \geqslant|B(z) R(z)|
$$

because $H(z)$ satisfies (10), and moreover $T_{22}(z)$ is BR. Finally, the right-side of (14) cannot be zero on the unit circle because $G(z)$ being stable, $Q(z)$ cannot have a zero on the unit circle. With the situation as outlined above, we can now apply an extended version of Rouche's theorem of complex-variable theory [3], stated below for convenience.

Rouche's theorem: If $f(z)$ and $g(z)$ are analytic interior to a simple closed Jordan curve $C$ and if they are continuous on $C$ and if the following conditions hold:

$$
\begin{aligned}
|f(z)| & \geqslant|g(z)|, \quad \text { for } z \text { on } C \\
f(z)+g(z) & \neq 0, \quad \text { for } z \text { on } C
\end{aligned}
$$

then the function $f(z)+g(z)$ has the same number of zeros inside $C$ as does $f(z)$.

If we take $C$ to be the unit circle, then we have the following conclusion:

Number of zeros of $Q(z)$ inside the unit circle

$$
\begin{aligned}
= & \text { Number of zeros of } A(z) \text { inside the unit circle } \\
& + \text { Number of zeros of } S(z) \text { inside the unit circle. }
\end{aligned}
$$

Thus

Number of zeros of $S(z)$ inside the unit circle $=m-n$.

Moreover, $H(z)$ is of degree $m-n$. Hence, it has no poles on or outside the unit circle. This proves the stability of $H(z)$. In conclusion, we have proved the following order-reduction theorem.

Theorem 1. Let $G(z)$ given by Eqn. (11) be BR with $q_{m} \neq 0$ and let $P(z)$ and $Q(z)$ be relatively prime. Let an LBR two-pair be extracted, and let the polynomial $A(z)$ be the LCM of the denominators of the transfer parameters $T_{i j}(z)$ of the extracted two-pair. Let $n$ denote the degree of $A(z)$. Let the remainder
$H(z)$ be of degree $m-n$ as in (12), with $s_{k} \neq 0$. Then $H(z)$ is necessarily $B R$.

Finally notice that, if $H(z)$ has degree $m-n$, then it is not only BR but also minimal. This is because, if $R(z)$ and $S(z)$ have cancelling factors, then the right side of (13) cannot make up the degree $m$ polynomials in $G(z)$.

\section{CONCLUDing REMARKS}

In our earlier paper [1], a number of first- and second-order LBR two-pair extractions have been tabulated. All of these are covered by Theorem 1 of this correspondence. As a result, the order-reduction approaches in [1] always lead to BR remainders, if we start from a BR function $G(z)$. However, Theorem 1 is more general and can be applied for higher order LBR two-pair extractions as well. The main application of the results of this paper is in the synthesis of low sensitivity digital filter structures that are free from zero input limit cycles. Many of the details can be found in [1], [2], [4].

\section{REFERENCES}

[1] P. P. Vaidyanathan and S. K. Mitra, "Low passband sensitivity digital filters: A generalized viewpoint and synthesis procedures," Proc. IEEE, vol. 72, 4, pp. 404-423, Apr. 1984.

[2] P. P. Vaidyanathan, "A general theory and synthesis procedure for low sensitivity digital filter structures," Ph.D. dissertation, Dep. Electrical and Computer Eng., Univ. of California, Santa Barbara, 1982.

[3] M. Marden, "Geometry of Polynomials," American Mathematical Society, RI, p. 5, 1966.

[4] P. P. Vaidyanathan and S. K. Mitra, "Passivity properties of low sensitivity digital filter structures," IEEE Trans. Circuits Syst., to be published.

\section{Diagnosability in the Decomposition Approach for Fault Location in Large Analog Networks}

T. OZAWA, J. W. BANDLER, AND A. E. SALAMA

Abstract-Diagnosability of analog networks using Salama, Starzyk, and Bandler's fault-location algorithm is investigated. A network is said to be $n$-diagnosable if, under the assumption that the network contains at most $n$ faulty subnetworks, all the faulty subnetworks can be located. A theorem for $n$-diagnosability of a network is derived.

Salama, Starzyk, and Bandler presented an efficient method to locate faults in an analog network by decomposition [1]. However, the diagnosability of a network by this approach, that is, whether or not all the faulty subnetworks can be located by this approach under the given measurement condition, was not clearly stated in [1]. This paper gives a solution to this problem.

Manuscript received September 25, 1984. This work was supported in part by the Ministry of Education, Science and Culture of Japan under Grant General Research: (C) 58550232 (1983).

T. Ozawa is with the Department of Electrical Engineering. Kyoto University, Kyoto, Japan.

J. W. Bandler is with the Department of Electrical and Computer Engineering, McMaster University, Hamilton, Ont., Canada.

A. E. Salama is with the Electronics and Communication Department, Cairo University, Giza, Egypt. 